\title{
Weil-Felix Test - A Diagnostic Tool for Rickettsial Diseases
}

\author{
Sanap SS, Thakur VA, Maniar J M, Vasave SV and \\ Vaidya SP* \\ Department of Clinical Pathology, Haffkine Institute for \\ Training, Research and Testing, India \\ *Corresponding author: Vaidya SP, Clinical Pathology \\ Department, Haffkine Institute for Training, Research \\ and Testing, Acharya Donde Marg, Parel, Mumbai \\ 400012, India
}

Received: December 05, 2016; Accepted: February 09, 2017; Published: February 14, 2017

\begin{abstract}
Rickettsial diseases are generally classified under the causes of Pyrexia of Unknown Origin (PUO) and high prevalence of this disease is found in developing countries like India. Diagnosis in such cases is difficult because early sign and symptoms are non-specific and varied. Mortality may be as high as $30-40 \%$ if untreated. Weil Felix in one of the cheapest screening methods available for laboratory diagnosis of rickettsial diseases but the major drawback of this method is its less sensitivity. A prospective testing was carried out in clinical pathology department in Haffkine Institute as a routine testing for the patients who are advised for extended Widal test. These patients come from the various government hospitals, private hospitals and tertiary care centres. The serum samples from 1464 PUO cases which included patients of all age group and from both government and private hospitals, were subjected to WeilFelix test (RK Diagnostics, Malad, Mumbai, India). The test was performed according to the manufacturer's instructions. Titers of $\geq 1: 80$ for OX K, OX 2 and OX 19 were considered significant. Of 1464 samples, rickettsial diseases were detected in $485(33.13 \%)$ samples. Seropositivity was higher among male subjects $262(54.02 \%)$ when compared with female subjects $223(45.98 \%)$ Most of the positive cases were in 5-10 age group. Prevalence of rickettsial diseases $485(33.13 \%)$ is significantly high, especially in children's and hence should be included in the differential diagnosis of PUO.
\end{abstract}

Keywords: Rickettsia; Weil Felix; Pyrexia of Unknown Origin (PUO); Scrub typhus; Rocky mountain scrub typhus; Tick borne scrub typhus

\section{Introduction}

Rickettsiae are characterised as group of small non-motile gram negative coccobacilli and are obligate intracellular organism. They are generally transmitted by arthropod vectors like lice, fleas and ticks [1]. Rickettsiae are globally prevalent and they have been report in all parts of India like Jammu and Kashmir, Himachal Pradesh, Uttaranchal, Rajasthan, Assam, West Bengal, Maharashtra, Kerala and Tamil Nadu [2]. Based on clinical manifestation species of Rickettsia can be categorized into spotted fever, typhus, and scrub typhus groups [3]. Rickettsial infections are attributed as one of the important causes of Pyrexia of Unknown Origin (PUO) and there is a need to differentiate it from other common febrile illnesses like enteric fever, malaria, dengue for appropriate treatment and better prophylaxis etc [4].

Clinical manifestation of Rickettsial infection have been very well documented but recent studies in asian countries have reported unusual complication associated with rickettsial infection such as gastrointestinal manifestations, tinnitus, and hepatitis syndromes [5]. Severities of this disease vary from self-limited illness to fulmination life threatening infection if untreated [6]. Mortality due to these infections is reported to occur in $1 \%$ to $30 \%$ of untreated cases $[7,8]$.

Treatment for rickettsial infection needs to started on the basis of clinical and epidemiological suspicion as no single laboratory finding is specific for early diagnosis, moreover the method used and sensitivity in testing this infection varies between with different laboratories. The greatest challenge to the clinician is the diagnostic dilemma posed by these infections early in their clinical course when antibiotic therapy is most effective. Micro-immunofluorescence, latex agglutination, indirect hemagglutination, immuno-peroxidase assay, and enzyme-linked immunosorbent assay are various serological tests available. Immunofluorescence Assay (IFA) is the "gold standard" technique [9]. Even though the sensitivity and specificity of Weil Felix test is low, in most of small laboratories, this method is the only one available. This study was conducted to know the prevalence of rickettsial diseases among various age group and gender wise prevalence of this infection.

\section{Materials and Methods}

Prospective study of 32 months from Jan 2014 to Aug 2016 was done in our clinical pathology department Haffkine Institute, Parel, India. Rickettsial diseases were diagnosed by Weil- Felix test (RK Diagnostics, Malad, Mumbai, India). Total of 1464 serum samples from PUO cases were subjected to Weil-Felix test. Test is based on the principle that some strains of Proteus share common somatic constituents with certain species of Rickettsia. Sera from patients infected with Rickettsia will, therefore, produce agglutination with Proteus antigen suspensions. Antigen suspension of Proteus OX 19 antigen reacts strongly with the sera of patients with typhus group rickettsiae and Rocky Mountain spotted fever, Proteus OX 2 with the sera of patients with spotted fever infections, while the Proteus OX K with the sera of patients infected with scrub typhus. Titers of $\geq 1: 80$ for OX 2, OX 19 and OX K were considered diagnostically significant. 


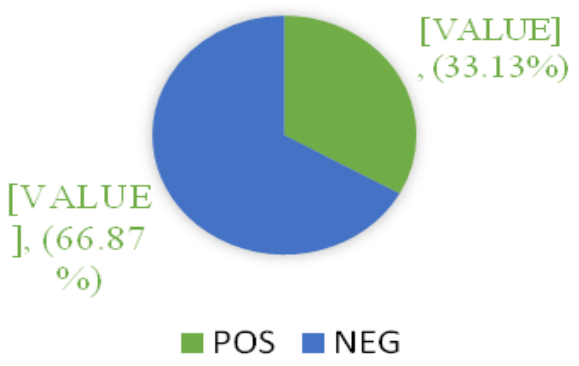

Figure 1: Total Puo Cases with their reactivity.

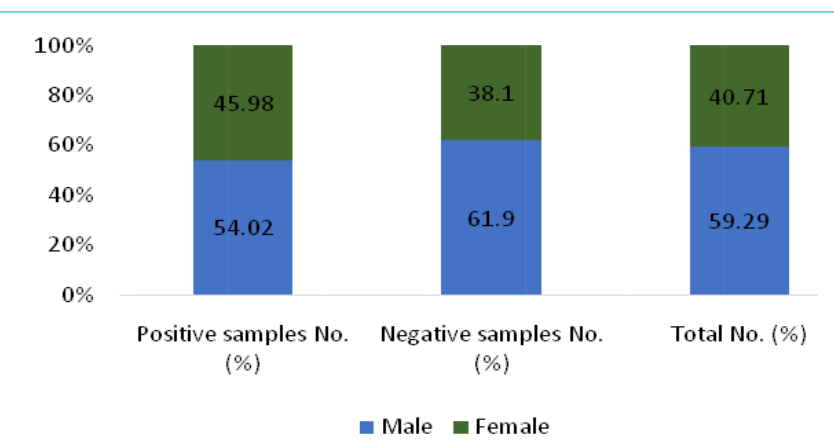

Figure 2: Gender -wise distribution of total cases.

\begin{tabular}{|c|c|c|c|}
\hline $\begin{array}{l}\infty \\
\infty \\
\infty\end{array}$ & $\begin{array}{l}\vec{F}= \\
\ddot{r}=\end{array}$ & 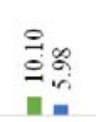 & $\begin{array}{l}6+a \\
-1\end{array}$ \\
\hline $0-5$ & $5-10$ & $10-15$ & $\begin{array}{c}\text { ABOVE } \\
15\end{array}$ \\
\hline
\end{tabular}

AGE (IN YEARS)

$=$ Male Positive samples No. $(\%) \quad$ memale Positive samples No. $(\%)$

Figure 3: Age-wise distribution of total positive cases.

\section{Results}

Of 1464 samples, rickettsial diseases were detected in 485 (33.13\%) samples. Seropositivity was higher among male subjects 262 (54.02\%) when compared with female subjects 223 (45.98\%). Most of the positive cases were in 5-10 age groups. 67 samples were reactive to OX 2 only, 166 to OX K only and 51 samples to OX 19 individually. When reactivity was seen towards two or more antigens in the same serum sample, combination of antigens demonstrated are OX $19+$ OX 2, OX $19+$ OX K, OX 2 + OX K and OX $19+$ OX 2 + OX K. In this combination OX $19+$ OX 2 was detected in 31 samples, OX $19+$ OX K in 47 samples, OX $19+$ OX $2+$ OX in 53 samples. Maximum reactivity was in combination OX $2+$ OX $\mathrm{K}$ which was detected in 70 samples. In the present study, rickettsial diseases were detected in $485(33.13 \%)$ samples with male predominance $262(54.02 \%)$ which is similar to previous studies [1,2]. Maximum reactivity was in combination OX $2+$ OX K. Recent studies have shown similar results [10]. Rickettsial infection is remerging with reports of this infection from different parts of worlds, predominantly in Asian countries [11]. The Rickettsial diseases are endemic in Southeast Asia, Northern Australia and pacific islands. Amongst Rickettisiosis scrub typhus is

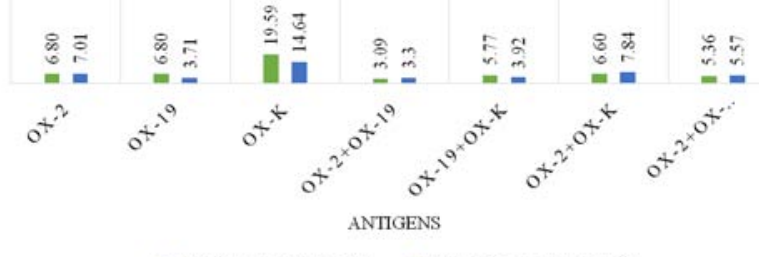

w Male Positive samples $(\%) \quad$ = Female Positive samples $(\%)$

Figure 4: Antigen-wise distribution of total positive cases.

Table 1: Total puo cases with their reactivity.

\begin{tabular}{|c|c|c|}
\hline Positive samples No. (\%) & Negative samples No. (\%) & Total No. (\%) \\
\hline $485(33.13 \%)$ & $979(66.87)$ & 1464 \\
\hline
\end{tabular}

Table 2: Gender -wise distribution of total cases.

\begin{tabular}{|c|c|c|c|}
\hline Gender & $\begin{array}{c}\text { Positive samples No. } \\
(\%)\end{array}$ & $\begin{array}{c}\text { Negative samples No. } \\
\text { (\%) }\end{array}$ & Total No. (\%) \\
\hline Male & $262(54.02)$ & $606(61.90)$ & $868(59.29)$ \\
\hline Female & $223(45.98)$ & $373(38.10)$ & $596(40.71)$ \\
\hline Total & $485(100)$ & $979(100)$ & $1464(100)$ \\
\hline
\end{tabular}

Table 3: Age-wise distribution of total positive cases.

\begin{tabular}{|c|c|c|c|}
\hline $\begin{array}{c}\text { Age- } \\
\text { Group } \\
\text { In Years }\end{array}$ & $\begin{array}{c}\text { Male Positive samples } \\
\text { No. (\%) }\end{array}$ & $\begin{array}{c}\text { Female Positive samples } \\
\text { No. (\%) }\end{array}$ & $\begin{array}{c}\text { Total } \\
\text { No. (\%) }\end{array}$ \\
\hline $0-5$ & $90(18.56)$ & $89(18.35)$ & $179(36.91)$ \\
\hline $5-10$ & $115(23.71)$ & $99(20.41)$ & $214(44.12)$ \\
\hline $10-15$ & $49(10.10)$ & $29(05.98)$ & $78(16.08)$ \\
\hline Above 15 & $8(01.65)$ & $6(01.24)$ & $14(2.89)$ \\
\hline Total & $262(54.02)$ & $223(45.98)$ & $485(100)$ \\
\hline
\end{tabular}

most common and is endemic in the tropical and subtropical regions of the Asian continent. The presence of rickettsial diseases in India has been documented in subhimalayan region of the country [12]. As per (Figure 1), out of 1464 samples, 485 (33.13\%) cases shows positive results. As per (Figure 2), out of total 1464 samples, $45.98 \%$ of female cases are showing positive results whereas $54.02 \%$ of male cases are showing positive results .As per above chart, it is seen that positive cases in males are more prominent than females. (Figure 3) shows the age wise distribution of positive cases. According to (Table 3), from the total number of sample received by our department, highest number of samples are from the age group of 5-10 in both male \& female, whereas less samples are from the age group of above 15 . Most of the cases which show positive test result for are belonging to the age group of 5-10. As per (Figure 4), from the total number of positive sample, maximum shows reactivity towards OX-K antigen $166(34.23 \%)$. Reactivity is maximum in combination of OX-2 + OX-K which is 70 (14.43\%).

\section{Discussion}

From the data obtained, it is clear that Seropositivity was higher among male subjects 262 (54.02\%) when compared with female subjects 223 (45.98\%).Males appear to be at higher risk to infection from tick-borne rickettsioses [13]. This is likely because of greater recreational or occupational exposures to tick habitats. However, in some spotted fever illnesses (e.g., Q fever), females seem to be less 
Table 4: Antigen-wise distribution of total positive cases.

\begin{tabular}{|c|c|c|}
\hline Antigens & Male Positive samples No. (\%) & Female Positive samples No. (\%) \\
\hline Only OX 2 & $33(06.80)$ & $34(07.01)$ \\
\hline Only OX 19 & $33(06.80)$ & $18(03.71)$ \\
\hline Only OX K & $95(19.59)$ & $71(14.64)$ \\
\hline OX 2 + OX 19 & $15(03.09)$ & $16(03.30)$ \\
\hline OX 19 + OX K & $28(05.77)$ & $19(03.92)$ \\
\hline OX 2+ OX K & $32(06.60)$ & $34(06.39)$ \\
\hline OX 2+ OX 19+ OX K & $26(05.36)$ & $47(9.69)$ \\
\hline Total & $262(54.02)$ & $70(14.43)$ \\
\hline
\end{tabular}

susceptible to the infection possibly due to a protective role of female hormones [14].

Most of the sample are reactive to OX-K antigen i.e.166 (34.23\%). $\mathrm{OX}-\mathrm{K}$ represents the scrub typhus infection. Because the vectors from which it is transmitted is present in most countries of the South-East Asia Region and it is endemic in certain geographical regions of India, Indonesia, Maldives, Myanmar, Nepal, Sri Lanka and Thailand [15].

Most of the positive cases were in 5-10 age groups. A thorough recreational and occupational history can help reveal potential exposures to tick habitats. In areas endemic for ticks, activities in commonplaces as playing in a backyard, visiting a neighbourhood park, gardening, or walking dogs are potential sources of tick exposure. Many types of environments serve as tick habitats, depending on the specific tick vector species. Areas with high uncut grass, weeds, and low brush might pose a high risk for certain vector species; however, these tick species also seek hosts in well-maintained grass lawns around suburban homes [16].

Weil-felix still remains an important tool for diagnosis of rickettsial infection, although rickettsiae can be isolated from or detected in clinical specimens using other serological techniques. New techniques such as micro Immunofluorescene Assay (IFA) have gained importance and have become reference test for detection of rickettsial infection. The procedure appears to be the most sensitive and specific method for the diagnosis of rickettsial infections. It is demonstrated that the sensitivity between WF test and micro IF results especially with a rise in WF titre is around 70\% [17]. Greater clinical awareness, a higher index of suspicion, better use of available diagnostic tools would increase the frequency with which rickettsial diseases are diagnosed [18].

Although rickettsiae can be isolated from or detected in clinical specimens, serological tests, especially Weil-Felix test, still remain an indispensable tool in diagnosis. In recent years the micro Immunofluorescene Assay (IFA) has become the reference test. The procedure appears to be the most appropriate for the diagnosis of rickettsial infections in low setup laboratories. Weil Felix test is the most established test to detect immune response of host to different Proteus antigens that cross-respond with rickettsiae. In spite of the fact that it needs specificity and sensitivity, it can be utilized as a primary diagnostic tool for detection of rickettsial infection in developing nations. One of the real restrictions of serology is the cross reactivity that frequently exists among antigens of pathogen inside similar class and at times in various genera. In reference research facilities, Cross-
Absorption (CA) procedures and Western blotting might be utilized to separate rickettsial infection by antibody evaluation, in developing nations like India where the study of disease transmission and burden of rickettsial disease is to a great extent.

Unfamiliar, high cost of directing examinations like immunofluorescence, Western blot or PCR based tests is extra obstacle in making exact conclusion of rickettsial illnesses. In such a case, straightforward, temperate Weil Felix test as beginning examination can guide a clinician in initiating proper treatment. Weil Felix test detects IgM antibody perceivable 5-10 days following the onset of disease indication. Entire cells of P. vulgaris OX 2 respond firmly with serum from individual tainted with Spotted Fever Group (SFG) rickettsiae except for those with Rocky Mountain Spotted Fever (RMSF); and entire cells of P. vulgaris OX 19 respond with serum from individual contaminated with typhus aggregate rickettsial and in addition with RMSF. Likewise, OX K strain of P. mirabilis agglutinates with serum from scrub typhus patients. For a test to be valuable in the finding of intense rickettsial contamination, the most critical criteria are sensitivity and the length of deferral between the onset and appearance of noticeable antibody titres. Cross-reaction or significant titre in double antigen can be determined by testing convalescent serum to validate significant increase in titre. Results of Weil Felix test along with other test like IgM antibody ELISA or immunofluorescence can improve the reliability of results barring cost per test for diagnostic purposes.

\section{Conclusion}

In conclusion, Weil Felix test can serve as primary test however not sole technique to perceive and analyze rickettsial diseases. In this manner, it is workable for most microbiology research facilities of the countries like India over to begin Weil Felix test, survey the load in their general vicinity and later on include other test like ELISA or immunofluorescence or Western blot contingent on the individual need and facilities accessible. Our outcomes demonstrate that rickettsial contaminations are one of the imperative reasons for PUO and dynamic observation of rickettsial disease is required to know correct extent and circulation of vector and diseases.

\section{References}

1. Raghu Kumar K, Tejashree P, Basavarajappa K. Serological evidence of rickettsial infections in and around Davangere. International Journal of Medical Science and Public Health. 2015; 4: 1731-1734.

2. Sood A, Chauhan1S. Rickettsial Diseases: An Urgent Need to Upgrade Diagnostic Facilities. International Journal of Recent Trends in Science and Technology. 2013; 7: 20-21. 
3. Tay S, Kamalanathan M, Rohani M. Detection of rickettsial antibodies using Weil-Felix (OXK and OX 19) antigens and the indirect immunoperoxidase assay. Southeast Asian Journal of Tropical Medicine \& Public Health. 2003 34: 171-174.

4. Mittal V, Gupta N, Bhattacharya D, Kumar K, Ichhpujani R, Singh S, et al Serological evidence of rickettsial infections in Delhi. The Indian journal of medical research. 2012; 135: 538 .

5. Nawab T, Srinivasa S, Reddy SP. A clinical study of rickettsial disease and its manifestations. Current Pediatric Research. 2015; 19.

6. Shenoy S, Baliga S, Ashok J. Rickettsial diseases in children. 2015

7. Rathi N, Rathi A. Rickettsial infections: Indian perspective. Indian pediatrics 2010; 47: 157-164

8. Prabhakaran A, Lal S, Biswas S, Vinoth S, Asraf AS, Mittal V. Serologica study of rickettsial diseases in human and rodent population in Chittoor dist (AP). 2010.

9. Mahajan S, Kashyap R, Kanga A, Sharma V, Prasher B, Pal L. Relevance of Weil-Felix test in diagnosis of scrub typhus in India. JAPI. 2006; 54: 619.

10. Ajantha GS, Patil SS, Chitharagi VB, Kulkarni RD. Rickettsiosis: a cause of acute febrile illness and value of Weil-Felix test. Indian journal of public health. 2013; $57: 182$

11. Munilakshmi P, Krishna MV, John MS, Deepa T, Avinash G, Reddy PS. FUO
Cases Showing Prevalence of Scrub Typhus: A Comparative Study by ELISA and Rapid Test in a Tertiary Care Hospital in Andhra Pradesh. India Int J Curr Microbiol App Sci. 2015; 4: 632-640

12. Sharma A, Mahajan S, Gupta ML, Kanga A, Sharma V. Investigation of an outbreak of scrub typhus in the Himalayan region of India. Japanese journal of infectious diseases. 2005; 58: 208.

13. Drexler NA, Dahlgren FS, Heitman KN, Massung RF, Paddock CD Behravesh CB. National surveillance of spotted fever group rickettsioses in the United States, 2008-2012. The American journal of tropical medicine and hygiene. 2016; 94: 26-34

14. Demeester R, Claus M, Hildebrand M, Vlieghe E, Bottieau E. Diversity of LifeThreatening Complications due to Mediterranean Spotted Fever in Returning Travelers. Journal of travel medicine. 2010; 17: 100-104.

15. Padbidri V, Gupta N. Rickettsiosis in India: a review. Journal of the Indian Medical Association. 1978; 71: 104-107.

16. FALCO RC, FISH D. Prevalence of Ixodes dammini near the homes of Lyme disease patients in Westchester County, New York. American Journal of Epidemiology. 1988; 127: 826-830.

17. Danave D, Kothadia S. Role of Weil Felix Test for Rickettsial Infections, IOSR Journal of Dental and Medical Sciences (IOSR-JDMS).1: 52-4.

18. Mahajan SK. Rickettsial diseases. J Assoc Physicians India. 2012; 60: 37-44.
Austin J Clin Pathol - Volume 4 Issue 1 - 2017

ISSN : 2381-9170 | www.austinpublishing group.com

Vaidya et al. (C) All rights are reserved
Citation: Sanap SS, Thakur VA, Maniar JM, Vasave SV and Vaidya SP. Weil-Felix Test - A Diagnostic Tool for Rickettsial Diseases. Austin J Clin Pathol. 2017; 4(1): 1046. 